\title{
Intelligent linkage analysis using gene density estimates
}

Sir - In November 1994, Antonarakis suggested in Nature Genetics ${ }^{1}$ that human genome linkage searches could be made more efficient by targeting gene-rich chromosomal regions. At that time, information about such regions was based largely on $\mathrm{CpG}$ island density, GC content and chromosome banding techniques, which provided only indirect evidence and very approximate locations. However, with the publication of the first human gene map ${ }^{2}$, it has become possible to pinpoint such regions more accurately and to relate them directly to the genetic map. By scanning the data available on the world wide web (http://www.ncbi.nlm.gov/science96), I have selected two sets of Généthon markers, set A and set B (Table 1), to screen expressed sequence tag (EST)-rich regions of the genome. within $5 \mathrm{cM}$ of at least $37 \%$ of the mapped ESTs. This figure is likely to be a substantial under-estimate, as I did not count ESTs mapping to overlapping intervals which extended further than $5 \mathrm{cM}$ from the markers. Also linkage can often be detected at distances much greater than 5 cM. It is interesting, within my own particular field of research, that set A markers would almost certainly have detected linkage to 10 of the 15 autosomal RP loci $^{3}$, while set $B$ markers would pick up a further three.

Variation in EST density is perhaps not as great as the 1994 data set reviewed by Antonarakis might have implied, but it is substantial never the less. Several chromosomes are conspicuous by their absence from the above lists. As Antonarakis ${ }^{1}$ pointed out, chromosomes

all listed as gene rich ${ }^{1}$, are all represented in the lists above.

This analysis is of course far from perfect. Genetic distances vary from map to map, so gene densities derived in this way must be regarded as estimates. Also these estimates use EST density rather than gene density, as the task of determining whether each of the 'unidentified transcripts' duplicates another entry would be immense. In time it may be possible to correlate each marker with the density of Unigene transcription units within a given genetic distance of it, to produce a Gene Density Index or GDI, which could be quoted along with heterozygosity for each marker, and used in the design of 'intelligent' linkage searches. Such searches would start with a list of candidate genes and regions then move on to a list of appropriately spaced markers ordered by gene density. This strategy would be equally applicable to genome scans for single gene defects or to searches for complex susceptibility or quantitative trait loci.

Nevertheless, sets $A$ and $B$ given above, used in conjunction with an appropriate list of candidate loci, should significantly reduce the necessity for wasted months spent 'fishing' for linkage. Those who reach

If each marker is assumed to screen the 10 -cM interval surrounding it, 40 markers cover approximately one tenth of a 4Morgan genome. Yet the markers listed in set $A$ lie within $5 \mathrm{cM}$ of at least 5,250 of the 20,104 ESTs mapped by Schuler and colleagues $^{2}$. Each marker in this set has within $5 \mathrm{cM}$ on either side of it an average of 125 ESTs, whereas one might expect a figure of around 50 ESTs per marker in a genome where EST distribution correlated with recombination frequency. Thus by searching one tenth of the genetic map, a researcher screens over a quarter of the localised ESTs. Use of set B covers a further 2,200 ESTs at 5-cM distance, with an average of 88 ESTs covered per marker. Use of both sets to screen approximately $17 \%$ of the genome would place markers
13 and 18 are relatively sparse in ESTs. Also chromosome 21, despite having a gene-rich long arm, appears unworthy of screening on the basis of the EST data. In contrast, chromosome 11 is EST rich with highly uneven distribution. Thus seven Généthon markers, covering less than $40 \%$ of this chromosome at the 5-cM level, account for just under $70 \%$ of the ESTs mapped to it. Chromosome 11 also boasts the most EST dense region, with 288 ESTs mapping in a $10-\mathrm{cM}$ region in the vicinity of $D 11 S 1313$. Predictably the HLA region on $6 p$ comes a close second with 237 ESTs in the $10 \mathrm{cM}$ around D6S273. In contrast, no ESTs have been assigned to the terminal $11 \mathrm{cM}$ of $17 \mathrm{q}$, and this on an otherwise relatively gene-rich chromosome. Chromosomes $1,9,15,16,17,19,20$ and 22 , the end of these lists without a 'bite' can at least derive some comfort from the knowledge that, once they find their locus, it will probably be in a relatively genepoor region, and thus the gene may prove easier to identify in the long run.

\section{Chris F. Inglehearn}

Department of Molecular Genetics, Institute of Ophthalmology, University College London, Bath Street, London, ECIV 9EL. e-mail: cinglehe@hgmp.mrc.ac.uk.

\section{Acknowledgements}

I thank the Wellcome Trust (Grant number 035535/2/92) for funding my research.

1. Antonarakis. S.E. Nature Genet. 8, 211-212 (1994) 2. Schuler, G.D. et al. Science 274, 540-546 (1996).

3. Sullivan, L.S. \& Daiger, S.P. Molec. Med. Today. 2, 380-386 (1996). 